\title{
sciendo
}

\section{CLINICAL AND SURGICAL ASPECTS OF MENINGOTHELIAL MENINGIOMA IN PRACTICE AND THE IMPLICATIONS OF BRAIN-IT SUMMER SCHOOL}

\author{
MIRCEA-VICENȚIU SĂCELEANU ${ }^{1}$, BOGDAN-NICOLAE ȚEPOSU ${ }^{2}$, ALINA-ADELA JOARZ $\breve{A}^{3}$, \\ ALEXANDRU BÎRSAN ${ }^{4}$, ADRIANA S S̆CELEANU ${ }^{5}$ \\ 1,2,3,4,5 “Lucian Blaga” University of Sibiu, Sibiu County Clinical Emergency Hospital
}

Keywords: meningioma, brain tumour, BrainIT, $3 D$ printing simulation, diagnostic certainty

\begin{abstract}
Mrs. F.M, a 77-year-old woman with prior diagnosis of Alzheimer disease, temporal lobe epilepsy, conversion disorder and essential familial hypercholesterolemia, presented for 2 months diffuse headache and vertigo, and in the past week, left brachial monoparesis and episodic amnesia become apparent, with progressive worsening and no improvement with medication therapy. The CT/MRI findings constituted a real challenge even for experienced practitioners. This article is hereby designed to meet the needs and necessities of young doctors within BrainIT international summer school.
\end{abstract}

\section{INTRODUCTION}

Meningioma is a benign tumour originating from the arachnoid, $90 \%$ being intracranial, the rest being able to develop at spinal level. It constitutes approximately $15 \%$ of all intracranial tumours and presents the most increased incidence in the seventh decade; it is rare in children but more common in women.(1)

Meningiomas are well-defined tumours and because of the origin in the arachnoid villosities, they can locate virtually anywhere, but most commonly in the Sylvian region, superior parasagittal surface of frontal and parietal lobes, sphenoid bone, tuberculum sellae, the upper surface of the cerebellum, cerebellopontine angle and the spinal cord.(2)

\section{CASE REPORT}

Mrs. F.M is a 77-year-old woman with Alzheimer disease, temporal lobe epilepsy, conversion disorder and essential hypercholesterolemia, who presents in the Emergency Room (ER) department in august 2021 with 2 months of diffuse headache and vertigo, and in the past week, left brachial monoparesis and episodic amnesia become apparent.

A brain CT scan is performed (figure no. 1), which outlines a right temporal tumour mass (possible glioblastoma multiforme) with significant peripheral edema and mass effect.

The radiologist recommends MRI with contrast to be performed, which highlighted a right temporo-parietal, corticosubcortical tumoral mass, with $51,7 / 40,4 / 48 \mathrm{~mm}$ (AP/LL/CC) in size, with gadolinium retention and restricted-diffusion pattern, areas of hemorrhagic necrosis with important vasogenic edema and mass effect. Once again, the radiologist raised the suspicion of a glioblastoma/astrocytoma (figure no. 2).

The patient agreed for the surgical removal of the tumour mass, and due to uncharacteristic and unclear CT/MRI aspect, a biopsy was performed, and the histopathological report showed the presence of a meningothelial meningioma WHO grade I.
Figure no. 1. Initial CT scan, performed in ER on the admission day $\left(24^{\text {th }}\right.$ of August $)$

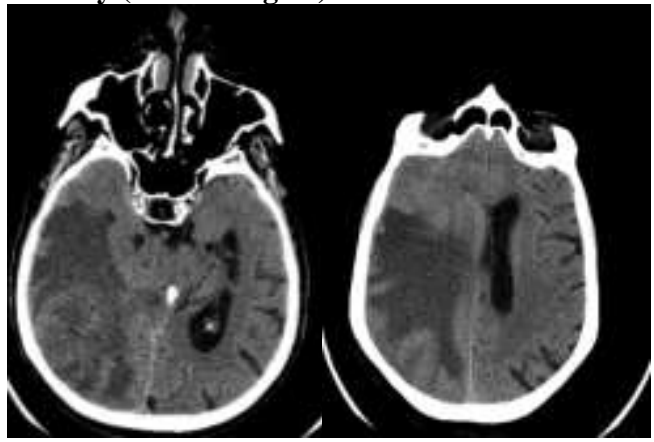

Figure no. 2. MRI exam (T2 axial and T2 sagittal with contrast), performed on $27^{\text {th }}$ of August

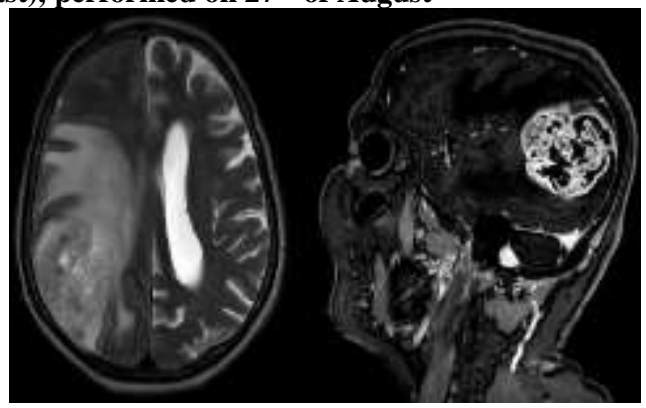

Postoperative evolution was satisfactory, no complications included, and the symptoms showed signs of withdrawing. The patient will be monitored closely to observe the evolution in the months that follow the surgical procedure.

This is a classic situation in which although the therapeutic conduct may be similar for different types of tumours (excision of the tumour mass), the per-se purpose/objective ends up being totally different:

${ }^{5}$ Corresponding author: Adriana Săceleanu, B-dul. Corneliu Coposu, Nr. 2-4, Sibiu, România, E-mail: teascu.adriana@yahoo.com, Phone: +40740 022931

Article received on 08.10.2021 and accepted for publication on 02.12.2021 


\section{CLINICAL ASPECTS}

curative/palliative with or without the risk of relapsing.

The average survival time from the appearance of the first symptom in cerebral astrocytomas is approximately of 67 months in brain astrocytomas and of 89 months in the cerebellum ones).(1)

Figure no. 3. CT scan $\left(1^{\text {st }}\right.$ of September $)-48 \mathrm{~h}$ after the surgical procedure

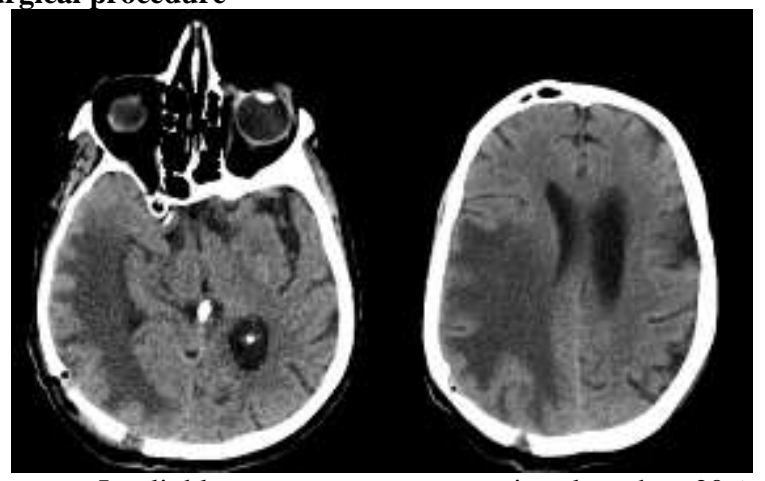

In glioblastoma tumour progression, less than $20 \%$ of patients survive 1 year after the onset of symptoms and only $10 \%$ more than two years; the prognosis is reserved. Treatment is unsatisfactory. The combination of neurosurgical excision, radiotherapy and anti-cancer medication seems to be the most advantageous, but survival beyond two years is exceptional. Therapeutic approach in the West is biopsy, pathological examination followed by radiotherapy and chemotherapy.(1)

On the other hand, meningothelial meningiomas (also known as syncytial or endothelial meningiomas) are the most common histological subtype of meningioma, found in $\sim 60 \%$ of all meningiomas, most frequently combined with fibrous meningioma $(40 \%)$ or in isolation $(17 \%)$. Furthermore, the tenyear survival rate for benign meningioma is $85 \%$, whereas for malignant meningiomas, the ten-years survival rate is approximately $62 \%$.(1)

Therefore, it is mandatory to highlight the importance of diagnostic certainty. Whereas it is known that the diagnostic certainty is established postoperatively with histopathological exam, surgical treatment is imperative, with rigorous technical criteria always being observed.

Such a situation is a challenge even for the experienced clinicians because the main interest is improving the patient's life quality, so taking vital decisions for the patient is needed, including during surgery.

Our University Centre makes sure that the young doctors are well trained but also takes care of the continuous improvement of the field specialists and, for this, the 2018-2021 BrainITsummer school, an international project, was conceived and developed as to respond to the needs of the students who want to have a career in Neurosurgery, allowing them to get relevant knowledge for their future workplace. Participating in this project, students will benefit from: improved practical skills in microsurgery and non-invasive techniques in neurosurgery, dexterity, good hand-eye coordination, excellent vision and visual-spatial awareness, good organizational ability, and communication skills, trained physical competences to cope with long hours and demanding nature of the work.

Besides offering a modern way of delivering specialist education, the programme will improve competences of students and specialists participating in the programme, as the remote part of the programme will be accompanied by 14 days trainee ships in a working hospital, where the course participants will get real life working experience accompanying the whole diagnostic process.

During the BrainIT project, certain simulations were performed, similar to neurosurgical procedures, using 3D models, including the procedure performed in the presented case.

In this type of procedure: removal of meningothelial meningioma, to mimic this tissue, the silicone samples were tested by neurosurgeons and the mixture Ecoflex 00-10 1:1 (A:B) was selected. Furthermore, this type of tumour usually has calcium deposits or calcification, which are small white spots that are more rigid than the tumour. Therefore, to reproduce these calcium deposits, the excess Smartfil@ PLA from other models (support material) was crushed into small pieces and added to the mixture. On the other hand, red pigment was added to improve the real character of the tumour.(3) In the following images, the models used can be observed:

Figure no. 4. 3D printed of the skull (a), the brain mold (b), the mold to define the working area inside the brain (c) and the tumour mold (d)
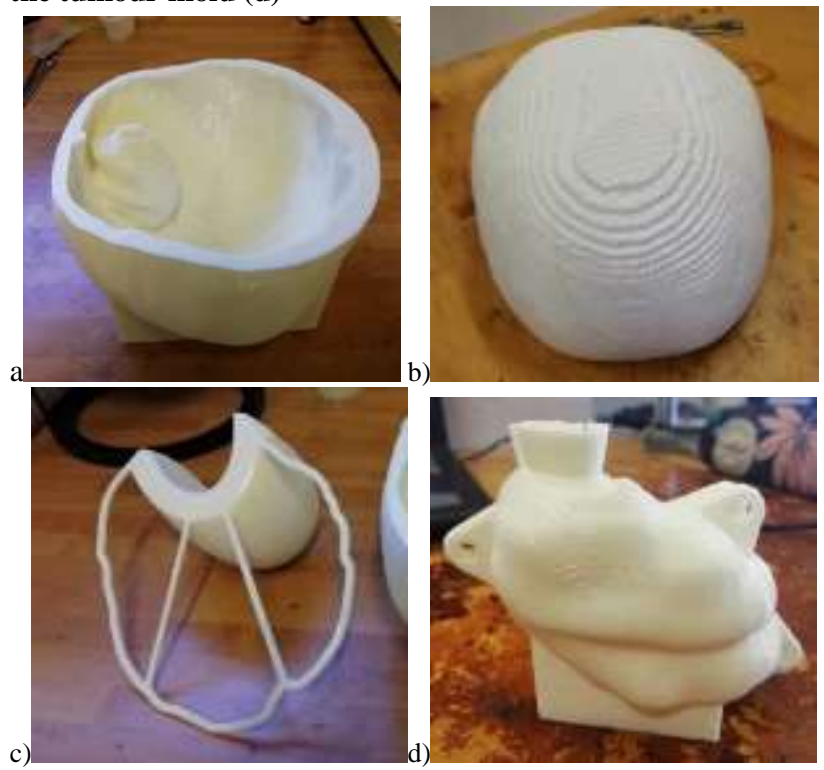

Figure no. 5. Casting of the different silicones in different proportions for the brain (a), tumour (b) and dura (c) models

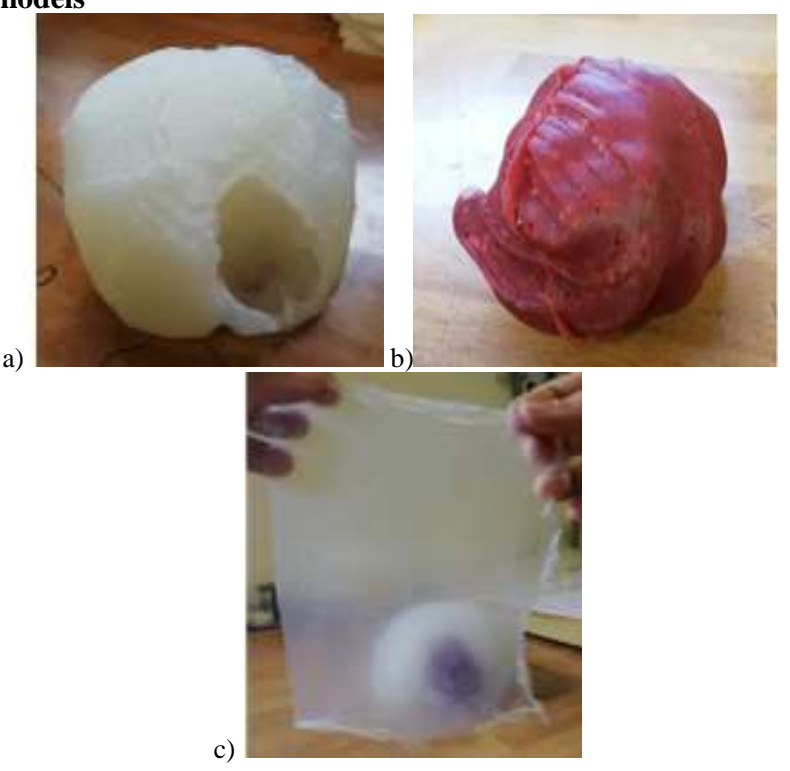

Once all the models were manufactured using AM (additive manufacturing, commonly known as 3D printing) and the silicone casting pours, the final assembly was carried out, as 


\section{CLINICAL ASPECTS}

shown in figure no. 6. Ten replicas of the model were produced and screwed on to the corresponding fixation mechanism, which allowed the desired orientation for the intervention. Finally, these models were used as training material in a Neuro-oncology workshop for medicine students.(3)

Figure no. 6. Final assembly of the model

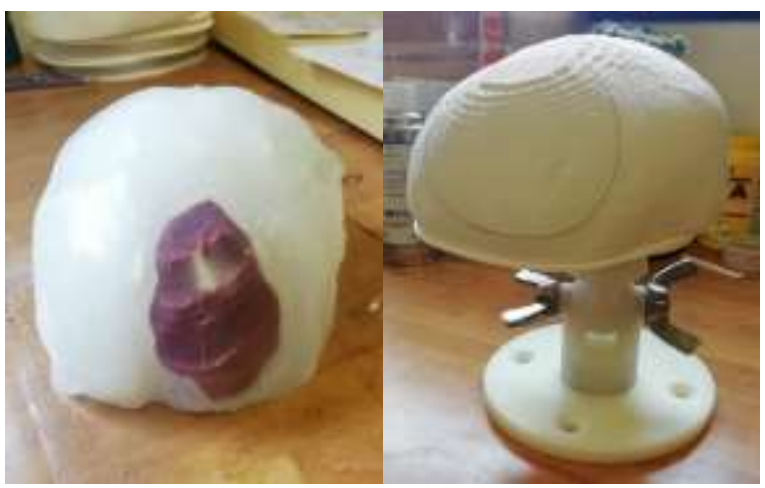

A simulation of the case presented above was produced, using the specific materials.

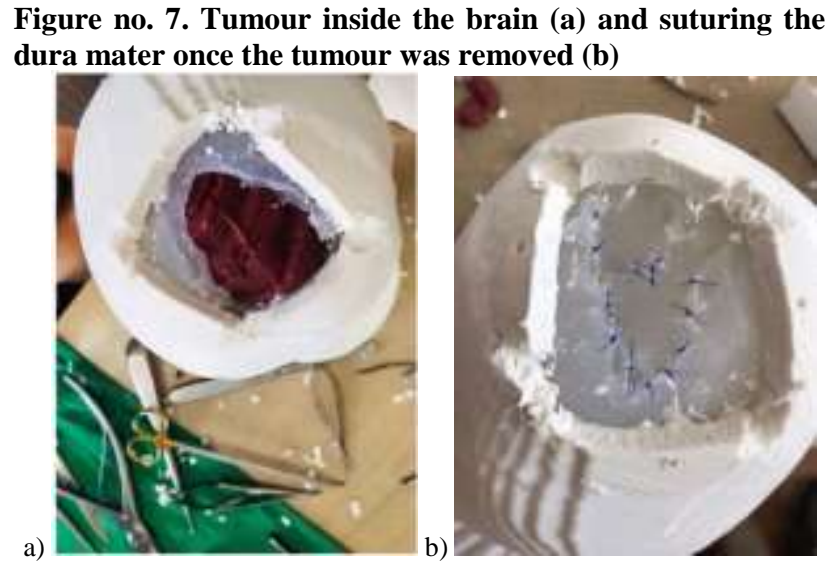

Figure no. 8. Final suture of the skull (a) with the tumour removed (b)

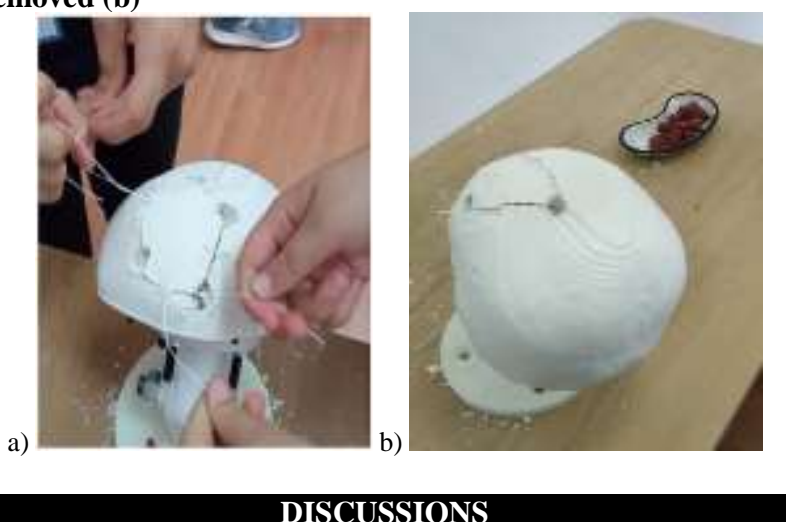

Meningiomas represent the commonest primary tumour of the central nervous system, accounting for around $38 \%$, and approximately half of them are benign.(4,5,6,7) This class of tumours originates from meningeal layers covering the brain or the spinal cord.(8) World Health Organization classifies meningiomas into three grades, with grade I being nonmalignant, type II - neither benign, nor malignant characteristics (they tend to become malignant in evolution), and type III malignant or anaplastic meningiomas. Genetic diseases, radiation, hormonal medication, obesity, alcoholism, some types of cancers or family history can be considered predisposing risk factors for developing meningiomas, but most of them are sporadic in origin.(9)

Clinical manifestations are different, depending on the tumour localisation within the brain or the spinal cord and certainly, depending on the tumour size, from patients having no symptoms to patients having several neurological deficits.(9)

Brain imaging plays the key role in diagnosing meningiomas and the brain magnetic resonance is the goldstandard investigation. Small-sized and asymptomatic meningiomas are usually observed in evolution with imaging techniques, whereas symptomatic or large-sized tumours are treated surgically (total surgical resection). $(4,10,11,12,13,14)$

Adjuvant radiotherapy after gross total resection of atypical meningiomas may decrease the risk for relapse and improve local control. $(15,16,17)$

Stereotactic radiosurgery (SRS) is another therapeutic method that can be used for patients who are unfit for surgery. SRS can be used for meningiomas of the skull base, recurrent, or incompletely resected meningiomas.(18)

The use of bevacizumab, a type of chemotherapy to target molecular alterations of vascular endothelial growth factor in patients with anaplastic meningiomas after surgical resection and radiotherapy, had shown successful results of tumour regression. $(19,20)$

The 5-year progression-free survival for grade 1 is $95.7 \%$, while the 10 -year progression-free survival for grade 1 is $90 \%$. Recurrence associated with the histological grade 1 has a $4.9 \%$ incidence of recurrence.(13)

\section{CONCLUSIONS}

Meningioma is a tumour that grows from the meningeal layers which cover and protect the brain and spinal cord. Most of these tumours are grade 1, which means that they are benign.

Some of the meningiomas do not produce symptoms, and many of them are discovered incidentally during imaging studies. A head CT scan or brain MRI is used to diagnose a meningioma.

The signs and symptoms depend on the location of the tumour in the brain. Symptoms such as headache, dizziness, visual impairments, weakness, convulsions, speech, or personality changes can occur.

There are no specific causes of meningioma, but certain risk factors have been identified.

A distinct aspect of the mentioned case is the suspicion raised of a brain glioblastoma or astrocytoma, both being tumours with high rate of morbidity/mortality, but also a high risk of recurrence. Therefore, the necessity of histopathological examination is outlined, for determining the precise diagnosis.

To determine the benefits of the BrainIT summer school, a statistics was obtained which showed that $90 \%$ of the students strongly considered the 3D models were useful to improve their skill in neurosurgery, $40 \%$ of them thought that this model was enough to feel the sensation of being in a real surgery; also, $100 \%$ considered this practical activity necessary in different sectors of medicine; $90 \%$ thought that the AM was an effective tool to support training and pre-clinical trial of professionals of medicine; $90 \%$ of the students were very satisfied with the practical activity with these $3 \mathrm{D}$ models.

Training in neurosurgery is a long-term process, which lasts in European countries for at least 6-7 years, necessary for learning the basic maneuvers, followed by continuous training throughout one's career. This long training process involves high costs for the health system and a higher risk in the medical 


\section{CLINICAL ASPECTS}

act. Because every new technical procedure that a surgeon performs will be done on a real patient.

From the perspective of neurosurgeons, this technique of real cases reconstruction and simulation of surgery brings important benefits. For resident physicians, it is a very good opportunity to practice basic surgical maneuvers before performing the procedure on the real patient. This greatly shortens the learning curve and at the same time the patient is treated with the utmost respect from an ethical point of view.

For senior neurosurgeons, it is a great opportunity to practice very complicated cases, which often have a very low incidence. That is why the role of surgery simulation is even greater.

\section{REFERENCES}

1. Săceleanu V. Neurochirurgie clinică. Sibiu: Editura Universității "Lucian Blaga" Sibiu; 2014.

2. Holleczek B, Zampella D, Urbschat S, Sahm F, von Deimling A, Oertel $\mathrm{J}$ et al. Incidence, mortality, and outcome of meningiomas: A population-based study from Germany. Cancer Epidemiology. 2019;62:101562.

3. Săceleanu V, Paz R, Garcia J, Rivero Y, Cindea CN, Cacciotti I, et al. Production of Synthetic Models for Neuro-oncology Training by Additive Manufacturing. Brain Sci. 2021;11, x.

4. Alruwaili AA, De Jesus O. Meningioma. StatPearls. Treasure Island (FL): StatPearls Publishing; 2021 Jan.

5. Ostrom QT, Cioffi G, Gittleman H, Patil N, Waite K, Kruchko K et al. CBTRUS Statistical Report: Primary Brain and Other Central Nervous System Tumors Diagnosed in the United States in 2012-2016, NeuroOncology. 2019; v1-v100.

6. Lim YS, Kim MK, Park BJ, Kim TS, Lim YJ. Long Term Clinical Outcomes of Malignant Meningiomas. The Korean Brain Tumor Society and The Korean Society for Neuro-Oncology. Seoul: Brain Tumor Res Treat.; 2013. Oct; 1(2): p. 85-90.

7. Wiemels J, Wrensch M, Claus E. Epidemiology and etiology of meningioma. J Neuro-oncology. 2010;Sep;99(3):307-14.

8. Louis DN, Perry A, Reifenberger G, vonDeimling A, Figarella-Branger D, Cavenee WK et al. The 2016 World Health Organization Classification of Tumors of the Central Nervous System: a summary. Acta neuropathologica. 2016;131(6):803-20.

9. Buerki RA, Horbisnky CM, Kruser T, Horowitz PM, James CD, Rimas VL. An overview of meningiomas. Future Oncology. 2018;14(21):2161-2177.

10. Huntoon K, Toland AMS, Dahiya S. Meningioma: A Review of Clinicopathological and Molecular Aspects. Front Oncology. 2020;10:579599.

11. Quddusi A, Shamim MS. Simpson grading as predictor of meningioma recurrence. J Pak Med Assoc. 2018;68(5):819-821.

12. Nanda A, Bir SC, Maiti TK, Konar SK, Missios S, Guthikonda B. Relevance of Simpson grading system and recurrence-free survival after surgery for World Health Organization Grade I meningioma. J Neurosurg. 2017;126(1):201-211.

13. Gousias K, Schramm J, Simon M. The Simpson grading revisited: aggressive surgery and its place in modern meningioma management. J Neurosurg. 2016;125(3):55160.

14. Bartek J Jr, Sjåvik K, Förander P, Solheim O, Gulati S, Weber $\mathrm{C}$ et al. 2. Predictors of severe complications in intracranial meningioma surgery: a population-based multicenter study. World Neurosurg. 2015;83(5):673-8.
15. Daraz B, Piela A. Treatment and prevention of EPHgestosis. Pieleg Polozna. 1978;(5):7-8

16. Johnson JE, Fuller SS, Endress MP, Rice VH. Altering patients' responses to surgery: an extension and replication. Research in nursing \& health. 1978;1(3):11121.

17. Aizer AA, Arvold ND, Catalano P, Claus EB, Golby AJ, Johnson MD et al. Adjuvant radiation therapy, local recurrence, and the need for salvage therapy in atypical meningioma. Neuro-oncology. 2014;16(11):1547-53.

18. Samblas J, Luis Lopez Guerra J, Bustos J, Angel Gutierrez-Diaz J, Wolski M, Peraza C et al. Stereotactic radiosurgery in patients with multiple intracranial meningiomas. J Buon. 2014;19(1):250-5.

19. Puchner MJ, Hans VH, Harati A, Lohmann F, Glas M, Herrlinger U. Bevacizumab-induced regression of anaplastic meningioma. Ann Oncol. 2010;21(12):24452446.

20. Aizer AA, Bi WL, Kandola MS, Lee EQ, Nayak L, Rinne ML et al. Extent of resection and overall survival for patients with atypical and malignant meningioma. Cancer. 2015;121(24):4376-81. 